\title{
Extinction Moment for a Branching Tree Evolution with Birth Rate and Death Rate Both Depending on Age
}

\author{
Xi Hu ${ }^{1},{ }^{1}$ Yun-Zhi Yan, ${ }^{2}$ Zhong-Tuan Zheng, ${ }^{1}$ Hong-Yan Li, ${ }^{3}$ and Hong-Yan Zhao ${ }^{1}$ \\ ${ }^{1}$ School of Mathematics, Physics and Statistics, Shanghai University of Engineering Science, Shanghai 201620, China \\ ${ }^{2}$ School of Statistics and Mathematics, Shanghai Lixin University of Accounting and Finance, Shanghai 201620, China \\ ${ }^{3}$ School of Management Studies, Shanghai University of Engineering Science, Shanghai 201620, China
}

Correspondence should be addressed to Xi Hu; xih_xih@163.com

Received 12 November 2020; Revised 26 December 2020; Accepted 28 January 2021; Published 11 February 2021

Academic Editor: Jiyuan Tao

Copyright (C) $2021 \mathrm{Xi} \mathrm{Hu}$ et al. This is an open access article distributed under the Creative Commons Attribution License, which permits unrestricted use, distribution, and reproduction in any medium, provided the original work is properly cited.

\begin{abstract}
In this paper, a branching tree evolution is established, in which the birth rate and the death rate are both dependent on node's age. The extinction probability and the t-pre-extinction (extinct before time $t$ ) probability are studied, by which the distribution of the extinction moment can be given. The analytical formula and the approximation algorithm for the distribution of extinction moment are given; furthermore, the analytical formula and the approximation algorithm of extinction probability are given, and a necessary and sufficient condition of extinction with probability 1 is given. It is the first time to study the distribution of extinction time for the branching process with birth rate and the death rate both depending on node's age, and the results will do great help in the theory of branching process. It is expected to be applied in the fields of biology, genetics, medicine, epidemiology, demography, nuclear physics, actuarial mathematics, algorithm, and data structures, etc.
\end{abstract}

\section{Introduction}

The classical biological reproduction model G-W branching process [1] has been extended to different biological reproduction models, such as branching processes in random environments [2-4] and branching population evolution models [5-11]. The age-dependent branching process was introduced by Bellman and Harris [6]. In branching models, the population extinction problem is one of the primary research contents. Many problems in branching models related to the population extinction are studied, but the distribution of extinction moment is hardly involved. In this paper, a branching tree evolution is established, in which the birth rate and death rate are both dependent on node's age. The extinction probability and the t-pre-extinction (extinct before time $t$ ) probability are studied, by which the distribution of the extinction moment can be given.

The paper is organized as follows. The model is described and the existence theorem is presented in Section 2. In Section 3, the extinction probability is studied, and the analytical formula and the approximation algorithm of the extinction probability are given. A necessary and sufficient condition of extinction with probability 1 is also given. In Section 4, the t-pre-extinction probability is studied, the iterative integral equation with unique solution is established, which is satisfied by the t-pre-extinction probability, and the analytical formula and the approximation algorithm of t-pre-extinction probability are given. The stochastic order of extinction moment is studied in Section 5. The conclusions are presented in Section 6.

\section{Description and Existence Theorem for the Model}

In this paper, based on the mechanism of asexual reproduction of biological population, a continuous time random graph evolution is constructed, in which a node's birth rate and death rate are both dependent on the node's age.

Given a population is composed of biological individuals (nodes). The evolution of the population is based on the following basic assumptions: 
(1) All nodes in the population are homogeneous and mutually independent

(2) The node's death rate in the population is a nonnegative function $\alpha(\cdot)$ dependent on the node's age, such that $\int_{0}^{\infty} \alpha(t) \mathrm{d} t=+\infty$

(3) The node's birth rate in the population is a nonnegative function $\beta(\cdot)$ dependent on the node's age

(4) Conditioned under a node being alive, the node's reproduction behaviors in the future are conditional independent

(5) Conditioned under a node being alive, the node's death is conditional independent with the node's reproduction

(6) At initial time $t=0$, there is only one initial node in the population (this condition is not essential, only for convenience of presentation)

(7) In addition to the initial node, each of other nodes in the population has only one parent node

Based on the above assumptions, the branching tree evolution is described as follows.

Given a node $i$ in the population, its age is $s$ at time $t$. For a sufficiently small period $\Delta t>0$, conditioned under node $i$ being alive at time $t$, the conditional probability for node $i$ being dead in the period $[t, t+\Delta t)$ is $\alpha(s) \Delta t+o(\Delta t)$, the conditional probability for node $i$ producing one child node in the period $[t, t+\Delta t)$ is $\beta(s) \Delta t+o(\Delta t)$, and the conditional probability for node $i$ producing more than one child node in the period $[t, t+\Delta t)$ is $o(\Delta t)$.

In the population, if node $j$ is a child of node $i$, then there is a directed link from node $i$ to node $j$. When at least one of the parent and child dies, the link between them is a virtual (dotted) line, and the dead node is called a virtual node. Otherwise, it is called a real node, and so on. At time $t \geq 0$, all nodes (real and virtual) and directed links (real and virtual) construct a directed random tree, denoted by $G_{t}(\cdot)$. And thus, the process of reproduction is an evolution of random trees, denoted by $\left\{G_{t}(\cdot)\right\}_{t \geq 0}$. As the evolution is characterized by the birth rate $\beta(\cdot)$ and the death rate $\alpha(\cdot)$, therefore, the model is referred to as "branching tree evolution with birth rate and death rate both depending on age," denoted by $\left\{G_{t}(\beta(\cdot), \alpha(\cdot))\right\}_{t \geq 0}$.

According to the definition of the model, $\forall t \geq 0$, the number of offspring born in period $(0, t]$ is finite, and no more than one offspring will be born at the same time. Therefore, the initial node and all its offspring nodes can be ordered as $1,2, \ldots, n, \ldots$ according to the order of birth time.

$$
\begin{aligned}
\forall n & \geq 1, \text { denote } \\
\vec{n} & =(1,2, \ldots, n), \\
\vec{f}(n) & =\left(f_{1}, f_{2}, \ldots, f_{n}\right): \quad f_{1}=0,1 \leq f_{k} \leq k-1,2 \leq k \leq n, \\
\vec{i}(n) & =\left(i_{1}, i_{2}, \ldots, i_{n}\right): \quad i_{k} \in\{0,1\}, 1 \leq k \leq n, \\
\vec{b}(n) & =\left(b_{1}, b_{2}, \ldots, b_{n}\right): \quad b_{i} \in R_{+}, 1 \leq i \leq n, \\
& \text { and } 0=b_{1}<b_{2}<\cdots<b_{n},
\end{aligned}
$$

where $\vec{n}$ is the vector of the labeled nodes; $\vec{f}(n)$ is the vector of the adjacency relation (parent-child relation) between nodes: $f_{1}=0$ means that the initial node has no parent node. $f_{k}=j, 1 \leq j \leq k-1$ indicates that node $j$ is the parent of node $k, 2 \leq k \leq n ; \vec{i}(n)$ is the vector of node's alivedeath status: $i_{k}=1$ denotes that node $k$ is alive and $i_{k}=0$ denotes that node $k$ is dead, $1 \leq k \leq n$; and $\vec{b}(n)$ is the birth time vector: $b_{1}=0$ represents there is an initial node at time $t=0, b_{j}$ is the time when node $j$ is born, $2 \leq j \leq n$, and $b_{1}<b_{2}<\cdots<b_{n}$ implies that no more than one node is born at the same time.

$\forall n \geq 1$, denote

$$
\begin{aligned}
\bar{F}_{n} & =\{\vec{f}(n)\}, \\
\bar{E}_{n} & =\{\vec{i}(n)\}, \\
\bar{B}_{n} & =\{\vec{b}(n)\}, \\
C_{3 \times n} & =(\vec{n}, \vec{f}(n), \vec{i}(n))^{T}, \\
S_{n}^{(3)} & =\{\vec{n}\} \times \bar{F}_{n} \times \bar{E}_{n}, \\
C_{4 \times n} & =\left(\vec{n}, \vec{f}(n), \vec{i}_{(n)}, \vec{b}(n)\right)^{T}, \\
S_{n} & =\{\vec{n}\} \times \bar{F}_{n} \times \bar{E}_{n} \times \bar{B}_{n}, \\
S & =\bigcup_{n=1}^{\infty} S_{n} .
\end{aligned}
$$$$
\forall C_{4 \times n}=(\vec{n}, \vec{f}(n), \vec{i}(n), \vec{b}(n))^{T}, \text { denote } I\left(C_{4 \times n}\right)=
$$
$\left\{k: i_{k}=1\right\} . \forall k \in I\left(C_{4 \times n}\right)$, denote

$$
\begin{aligned}
& D_{k}\left(C_{4 \times n}\right)=\left\{C_{4 \times(n+1)}=(\overrightarrow{n+1}, \vec{f}(n+1), \vec{r}(n+1), \vec{b}(n+1))^{T}:\right. \\
& \left.\vec{b}(n+1)=\left(\vec{b}(n), b_{n+1}\right) ; \vec{f}(n+1)=\left(\vec{f}(n), f_{n+1}\right), f_{n+1}=k ; r_{j} \leq i_{j}, 1 \leq j \leq n\right\} .
\end{aligned}
$$


$\forall C_{4 \times n}=(\vec{n}, \vec{f}(n), \vec{i}(n), \vec{b}(n))$, and $b_{n} \leq s<t$, define the function $f_{n}^{(k)}\left(s, t, C_{4 \times(n+1)} \mid C_{4 \times n}\right)$ on $S_{n+1}$. $\forall C_{4 \times(n+1)}=(\overrightarrow{n+1}, \vec{f}(n+1), \vec{r}(n+1), \vec{b}(n+1))$,

$$
\begin{aligned}
f_{n}^{(k)}\left(s, t, C_{4 \times(n+1)} \mid C_{4 \times n}\right)= & \widetilde{I}_{D_{k}\left(C_{4 \times n}\right)}\left(C_{4 \times(n+1)}\right)\left(\prod_{\substack{j \in I\left(C_{4 \times n}\right) \\
j \neq k, r_{j}=0}} \int_{s}^{t} e^{-\int_{s-b_{j}}^{y-b_{j}} \beta(u) \mathrm{d} u} \alpha(y) e^{-\int_{s}^{y} \alpha(u) \mathrm{d} u} \mathrm{~d} y\right) \\
& \times\left(\prod_{j \in I\left(C_{4 \times n}\right)} e^{-\int_{j \neq k, r_{j}=1}^{t-b_{j}} \beta(u) \mathrm{d} u} e^{-\int_{s}^{t} \alpha(u) \mathrm{d} u}\right) \\
& \times \beta\left(b_{n+1}-b_{k}\right) g_{1}\left(t, b_{k}, r_{k}\right) g_{2}\left(t, b_{n+1}, r_{n+1}\right),
\end{aligned}
$$

where $\widetilde{I}_{A}(\cdot)$ is a indicative function, and

$$
\begin{aligned}
& g_{1}\left(t, b_{k}, r_{k}\right)=\left\{\begin{array}{l}
e^{-\int_{s}^{t} \alpha(u) \mathrm{d} u} e^{-\int_{s-b_{j}}^{t-b_{j}} \beta(u) \mathrm{d} u}, \quad r_{k}=1, \\
e^{-\int_{s-b_{k}}^{b_{n+1}-b_{k}} \beta(u) \mathrm{d} u} \int_{b_{n+1}}^{t} e^{-\int_{b_{n+1}-b_{k}}^{y-b_{k}} \beta(u) \mathrm{d} u} \alpha(y) e^{-\int_{s}^{y} \alpha(u) \mathrm{d} u} \mathrm{~d} y, \quad r_{k}=0,
\end{array}\right. \\
& g_{2}\left(t, b_{k}, r_{n+1}\right)=\left\{\begin{array}{l}
e^{-\int_{b_{n+1}}^{t} \alpha(u) \mathrm{d} u} e^{-\int_{b_{n+1}}^{t} \beta(u) \mathrm{d} u}, \quad r_{n+1}=1, \\
\int_{b_{n+1}}^{t} e^{-\int_{b_{n+1}}^{y} \beta(u) \mathrm{d} u} \alpha(y) e^{-\int_{b_{n+1}}^{y} \alpha(u) \mathrm{d} u} \mathrm{~d} y, \quad r_{n+1}=0 .
\end{array}\right.
\end{aligned}
$$

$\forall C_{4 \times n}=(\vec{n}, \vec{f}(n), \vec{i}(n), \vec{b}(n))$, and $b_{n} \leq s<t$, define the function $f_{n}\left(s, t, C_{4 \times(n+1)} \mid C_{4 \times n}\right)$ on $S_{n+1}$ :

$$
f_{n}\left(s, t, C_{4 \times(n+1)} \mid C_{4 \times n}\right)=\sum_{k \in I\left(C_{4 \times n}\right)} f_{n}^{(k)}\left(s, t, C_{4 \times(n+1)} \mid C_{4 \times n}\right) .
$$

Let $\mathrm{d} b_{n+1}$ be a Lebesgue measure on $(s, t]$, for a given $\vec{b}(n)$, and $b_{n} \leq s$, then $\delta_{\{\vec{b}(n)\}} \times \mathrm{d} b_{n+1}$ is a measure on $\left(\bar{B}_{n+1}, \mathscr{B}\left(\bar{B}_{n+1}\right)\right)$. Let $\mu_{n+1}(\cdot)$ be a count measure on $\left(S_{n+1}^{(3)}, \mathscr{B}\left(S_{n+1}^{(3)}\right)\right)$, denote $v_{n+1}(\cdot)=\mu_{n+1} \times\left(\delta_{\{\vec{b}(n)} \times \mathrm{d} b_{n+1}\right)$ $(\cdot)$, and then $v_{n+1}(\cdot)$ is a measure on $\left(S_{n+1}, \mathscr{B}\left(S_{n+1}\right)\right)$. Define $\forall D_{n+1} \in \mathscr{B}\left(S_{n+1}\right)$,

$$
\begin{aligned}
& Q_{n}\left(s, t, D_{n+1} \mid C_{4 \times n}\right)=\int_{D_{n+1}} f_{n}\left(s, t, C_{4 \times(n+1)} \mid C_{4 \times n}\right) v_{n+1} \\
& \quad\left(\mathrm{~d} C_{4 \times(n+1)}\right) .
\end{aligned}
$$

Then, $\forall 0 \leq s<t, C_{4 \times n} \in S_{n}, Q_{n}\left(s, t, \cdot \mid C_{4 \times n}\right)$ is a measure on $\quad\left(S_{n+1}, \mathscr{B}\left(S_{n+1}\right)\right), \quad \forall 0 \leq s<t, \quad D_{n+1} \in \mathscr{B}\left(S_{n+1}\right)$, $Q_{n}\left(s, t, D_{n+1} \mid \cdot\right)$ is a measurable function on $\left(S_{n}, \mathscr{B}\left(S_{n}\right)\right)$. Let

$$
\begin{aligned}
A_{2 \times n} & =\left(\begin{array}{c}
\vec{n} \\
\vec{f}(n)
\end{array}\right), \\
B_{2 \times n} & =\left(\begin{array}{c}
\vec{i}(n) \\
\vec{b}(n)
\end{array}\right), \\
C_{4 \times n} & =\left(\begin{array}{c}
A_{2 \times n} \\
B_{2 \times n}
\end{array}\right) .
\end{aligned}
$$

Let $N(t)$ be the number of nodes in the random branching tree $G_{t}(\beta(\cdot), \alpha(\cdot))$, then $G_{t}(\beta(\cdot), \alpha(\cdot))$ can be expressed by a $2 \times N(t)$ matrix, i.e.,

$$
G_{t}(\beta(\cdot), \alpha(\cdot))=A_{2 \times N(t)} .
$$


The birth time and the alive-death status of the $N(t)$ nodes in $G_{t}(\beta(\cdot), \alpha(\cdot))$ can be expressed by the $2 \times N(t)$ matrix $B_{2 \times N(t)}$, and denote

$$
X_{t}=\left(\begin{array}{c}
A_{2 \times N(t)} \\
B_{2 \times N(t)}
\end{array}\right)=C_{4 \times N(t)} .
$$

We have the following theorem.

Theorem (existence) $\left\{G_{t}(\beta(\cdot), \alpha(\cdot))\right\}_{t \geq 0}$ is the marginal process of the nonhomogeneous Markov process $\left\{X_{t}\right\}_{t>0}$ in the state space $S$, where the transfer function of $\left\{X_{t}\right\}_{t \geq 0}$ is $\forall D \in \mathscr{B} \quad(S), 0 \leq s<t, n \geq 1, C_{4 \times n}=(\vec{n}, \vec{f} \quad(n), \vec{i}(n), \vec{b}$ $(n))^{T} \in S_{n}$, here $D_{m} \in \mathscr{B}\left(S_{m}\right)$

$$
\begin{aligned}
& P\left(X(t) \in D \mid X(s)=C_{4 \times n}\right) \\
& =\sum_{m=1}^{\infty} P\left(X(t) \in D, N(t)=m \mid X(s)=C_{4 \times n}\right) \\
& =\sum_{m=1}^{\infty} P\left(X(t) \in D_{m} \mid X(t)=C_{4 \times n}\right) \\
& =P\left(X(t) \in D_{n} \mid X(s)=C_{4 \times n}\right)+\sum_{m=n+1}^{\infty} P\left(X(t) \in D_{m} \mid X(s)=C_{4 \times n}\right),
\end{aligned}
$$

where

$$
\begin{aligned}
P\left(X(t) \in D_{n} \mid X(s)=C_{4 \times n}\right)= & \sum_{\vec{r}(n) \in \hat{D}_{n}} \prod_{\substack{j \in I \\
r_{j}=0}} \int_{s}^{t} e^{-\left[\lambda\left(y-b_{j}\right)-\lambda\left(s-b_{j}\right)\right]} \alpha e^{-\alpha(y-s)} \mathrm{d} y \times \prod_{j \in I\left(C_{4 \times n}\right)} e^{-\left[\lambda\left(t-b_{j}\right)-\lambda\left(s-b_{j}\right)\right]} e^{-\alpha(t-s)}, \\
P & \left.X(t) \in D_{n+k} \mid X(s)=C_{4 \times n}\right)=P\left(X(t) \in D_{n+k} \mid X(s)=C_{4 \times n}\right) \\
= & \int_{S_{n+1}} \cdots \int_{S_{n+k-1}} \int_{D_{n+k}} Q_{n}\left(s, b_{n+1}, \mathrm{~d} C_{4 \times(n+1)} \mid C_{4 \times n}\right) \cdots \\
& \times Q_{n+k-2}\left(b_{n+k-2}, b_{n+k-1}, d C_{4 \times(n+k-1)} \mid C_{4 \times(n+k-2)}\right) \\
& \times Q_{n+k-1}\left(b_{n+k-1}, t, \mathrm{~d} C_{4 \times(n+k)} \mid C_{4 \times(n+k-1)}\right), \quad k \geq 1
\end{aligned}
$$

where $\quad \widehat{D}_{n}=\left\{\vec{r}(n):(\vec{n}, \vec{f}(n), \vec{r}(n), \vec{b}(n)) \in D_{n}\right\}$. Substituting (12) and (13) into (11), the transfer function of $\left\{X_{t}\right\}_{t \geq 0}$ is obtained.

It is not difficult to prove that $S$ is a Borel subset of the separable complete distance space $R^{\infty}$, and the existence theorem of $\left\{X_{t}\right\}_{t \geq 0}$ can be proved by the existence theorem of Markov process. $\left\{G_{t}(\beta(\cdot), \alpha(\cdot))\right\}_{t \geq 0}$ is a marginal process of $\left\{X_{t}\right\}_{t \geq 0}$, and thus, the existence of $\left\{G_{t}(\beta(\cdot), \alpha(\cdot))\right\}_{t \geq 0}$ is proved.

\section{The Extinction Probability}

Define

$$
\begin{aligned}
T(\omega) & =\inf \{t>0: \text { population extinction in the period }[0, t)\} \\
P(t) & =P\{T(\omega) \leq t\}, \quad 0 \leq t<\infty \\
P(\infty) & =P\{T(\omega)<\infty\} .
\end{aligned}
$$

$T(\omega)$ is called the extinction moment, at which the population extinct. The probability $P(t)$ is called t-preextinction probability, which is the probability of the population extinct before time $t$, and the probability $P(\infty)$ is called extinction probability. The distribution of extinction moment $T(\omega)$ is given by t-pre-extinction probability and $P(T(\omega)=\infty)=1-P(T(\omega)<\infty)=1-P(\infty)$. If $P(\infty)=1$, i.e., $P(T(\omega)=\infty)=0$. Then, $T(\omega)$ is a realvalued random variable, so the t-pre-extinction probability $P(t), t \geq 0$ is the distribution function of $T(\omega)$.

In this section, the extinction probability for the branching tree evolution $\left\{G_{t}(\beta(\cdot), \alpha(\cdot))\right\}_{t \geq 0}$ is studied, the analytical formula and the approximation algorithm of extinction probability are given, and a necessary and sufficient condition of extinction with probability 1 is also given.

Let $\eta(t)$ be the number of nodes that are alive in the population at time $t$, then $P(t)=P(\eta(t)=0), 0 \leq t<\infty$. Obviously, $\quad\{\eta(s)=0\} \subseteq\{\eta(t)=0\} \quad$ when $\quad s \leq t$, and $P(\infty)=\lim _{t \longrightarrow \infty} P(\eta(t)=0)$. 
It is obvious that $P(t)$ has the following properties:

(1) $\forall 0<s<t, 0=P(0)<P(s)<P(t)<P(\infty)$

(2) $P(t)$ is continuous on $[0, \infty)$

Lemma 1. Given a node $i$ in the population, its lifespan is $Y$, and then $Y$ has the probability density function:

$$
f_{Y}(t)=\left\{\begin{array}{cl}
\alpha(t) \mathrm{e}^{-\int_{0}^{t} \alpha(u) \mathrm{d} u}, & t \geq 0, \\
0, & t<0,
\end{array}\right.
$$

where $\alpha(\cdot)$ is the node's death rate.

It is easy to prove Lemma 1.

Theorem 1. The following iterative integral equation is satisfied by the t-pre-extinction probability $P(t), 0<t<\infty$

$$
\begin{aligned}
P(t)= & \int_{0}^{t} e^{-\int_{0}^{s}(1-P(t-u)) \beta(u) \mathrm{d} u} \\
& \cdot \alpha(s) e^{-\int_{0}^{s} \alpha(u) \mathrm{d} u} \mathrm{~d} s, \quad 0<t<\infty,
\end{aligned}
$$

where $\beta(\cdot)$ is the node's birth rate and $\alpha(\cdot)$ is the node's death rate.
Proof. $\forall 0<t<\infty, 0<P(t)<1$, Let $Y$ be the node's lifespan, then we get

$$
\begin{aligned}
P(t) & =P(\eta(t)=0)=P(\eta(t)=0, Y \leq t) \\
& =\int_{0}^{t} P(\eta(t)=0 \mid Y=s) \cdot \alpha(s) e^{-\int_{0}^{s} \alpha(u) \mathrm{d} u} \mathrm{~d} s .
\end{aligned}
$$

In the following, we first calculate $P(\eta(t)=0 \mid Y=s)$. Equally divide the interval $[0, s]$ into $n$ intervals, denote $\delta=(s / n)$, and let $A_{k}$ be the random event: the initial node produces a child node in the period $(k \delta,(k+1) \delta]$ and the offspring of this child node extinct before time $t$ or the initial node does not produce a child node in the period $(k \delta,(k+1) \delta], 0 \leq k \leq n-1$.

When $\delta$ is sufficiently small, the probability that the initial node does not produce a child node in the period $(k \delta,(k+1) \delta]$ is $1-\beta(k \delta) \cdot \delta+o(\delta)$; the probability of producing more than one child node is $o(\delta)$; the probability of producing one child node is $\beta(k \delta) \cdot \delta+o(\delta)$, and this child node's offspring extinct before time $t$ with probability $P(t-k \delta), 0 \leq k \leq n-1$, so

$$
\begin{aligned}
P\left(A_{k} \mid Y=s\right)= & \beta(k \delta) \cdot \delta \cdot P(t-k \delta)+1-\beta(k \delta) \\
& \cdot \delta+o(\delta), \quad 0 \leq k \leq n-1 .
\end{aligned}
$$
have

Noting the independent assumptions of the model, we

$$
\begin{aligned}
P(\eta(t) & =0 \mid Y=s)=\lim _{\delta \longrightarrow 0} P\left(\bigcap_{k=0}^{n-1} A_{k} \mid Y=s\right)=\lim _{\delta \longrightarrow 0} \prod_{k=0}^{n-1} P\left(A_{k} \mid Y=s\right) \\
& =\lim _{\delta \longrightarrow 0} \prod_{k=0}^{n-1}[\beta(k \delta) \cdot \delta \cdot P(t-k \delta)+(1-\beta(k \delta) \cdot \delta)+o(\delta)] \\
& =\lim _{\delta \longrightarrow 0} \prod_{k=0}^{n-1}[1-\beta(k \delta) \cdot(1-P(t-k \delta)) \cdot \delta+o(\delta)] .
\end{aligned}
$$

Then,

$$
\begin{aligned}
\ln P(\eta(t) & =0 \mid Y=s)=\lim _{\delta \longrightarrow 0} \sum_{k=0}^{n-1} \ln [1-\beta(k \delta) \cdot(1-P(t-k \delta)) \cdot \delta+o(\delta)] \\
& =-\lim _{\delta \longrightarrow 0} \sum_{k=0}^{n-1} \beta(k \delta) \cdot(1-P(t-k \delta)) \cdot \delta+o(\delta) .
\end{aligned}
$$

That is

$$
P(t)=\int_{0}^{t} e^{-\int_{0}^{s}(1-P(t-u)) \beta(u) \mathrm{d} u} \cdot \alpha(s) e^{-\int_{0}^{s} \alpha(u) \mathrm{d} u} \mathrm{~d} s .
$$

$$
P(\eta(t)=0 \mid Y=s)=e^{-\int_{0}^{s}(1-P(t-u)) \beta(u) \mathrm{d} u} .
$$

Thus, the theorem is proved.

Denote

So 


$$
g(x)=\int_{0}^{\infty} e^{-\int_{0}^{s}(1-x) \beta(u) \mathrm{d} u} \cdot \alpha(s) e^{-\int_{0}^{s} \alpha(u) \mathrm{d} u} \mathrm{~d} s, \quad 0 \leq x \leq 1 . \quad(23) \quad x=g(x), \quad 0 \leq x \leq 1
$$

Lemma 2. The extinction probability $P(\infty)$ is a solution of Proof. $0<t_{0}<t<\infty$, the equation

$$
\begin{aligned}
& |P(t)-g(P(\infty))| \\
& =\left|\int_{0}^{t} e^{-\int_{0}^{s}(1-P(t-u)) \beta(u) \mathrm{d} u} \cdot \alpha(s) e^{-\int_{0}^{s} \alpha(u) \mathrm{d} u} \mathrm{~d} s-\int_{0}^{\infty} e^{-\int_{0}^{s}(1-P(\infty)) \beta(u) \mathrm{d} u} \cdot \alpha(s) e^{-\int_{0}^{s} \alpha(u) \mathrm{d} u} \mathrm{~d} s\right| \\
& \leq\left|\int_{0}^{t_{0}} e^{-\int_{0}^{s}(1-P(t-u)) \beta(u) \mathrm{d} u} \cdot \alpha(s) e^{-\int_{0}^{s} \alpha(u) \mathrm{d} u} \mathrm{~d} s-\int_{0}^{t_{0}} e^{-\int_{0}^{s}(1-P(\infty)) \beta(u) \mathrm{d} u} \cdot \alpha(s) e^{-\int_{0}^{s} \alpha(u) \mathrm{d} u} \mathrm{~d} s\right| \\
& \quad+\left|\int_{t_{0}}^{t} e^{-\int_{0}^{s}(1-P(t-u)) \beta(u) \mathrm{d} u} \cdot \alpha(s) e^{-\int_{0}^{s} \alpha(u) \mathrm{d} u} \mathrm{~d} s-\int_{t_{0}}^{t} e^{-\int_{0}^{s}(1-P(\infty)) \beta(u) \mathrm{d} u} \cdot \alpha(s) e^{-\int_{0}^{s} \alpha(u) \mathrm{d} u} \mathrm{~d} s\right| \\
& \quad+\int_{t}^{\infty} e^{-\int_{0}^{s}(1-P(t-u)) \beta(u) \mathrm{d} u} \cdot \alpha(s) e^{-\int_{0}^{s} \alpha(u) \mathrm{d} u} \mathrm{~d} s,
\end{aligned}
$$

$\forall \varepsilon>0, \exists t_{0}, 0<t_{0}<t$, and let $t$ be large enough, such that

$$
\begin{aligned}
& \left|\int_{0}^{t_{0}} e^{-\int_{0}^{s}(1-P(t-u)) \beta(u) \mathrm{d} u} \cdot \alpha(s) e^{-\int_{0}^{s} \alpha(u) \mathrm{d} u} \mathrm{~d} s-\int_{0}^{t_{0}} e^{-\int_{0}^{s}(1-P(\infty)) \beta(u) \mathrm{d} u} \cdot \alpha(s) e^{-\int_{0}^{s} \alpha(u) \mathrm{d} u} \mathrm{~d} s\right|<\frac{\varepsilon}{3}, \\
& \left|\int_{t_{0}}^{t} e^{-\int_{0}^{s}(1-P(t-u)) \beta(u) \mathrm{d} u} \cdot \alpha(s) e^{-\int_{0}^{s} \alpha(u) \mathrm{d} u} \mathrm{~d} s-\int_{t_{0}}^{t} e^{-\int_{0}^{s}(1-P(\infty)) \beta(u) \mathrm{d} u} \cdot \alpha(s) e^{-\int_{0}^{s} \alpha(u) \mathrm{d} u} \mathrm{~d} s\right|<\frac{\varepsilon}{3}, \\
& \int_{t}^{\infty} e^{-\int_{0}^{s}(1-P(\infty)) \beta(u) \mathrm{d} u} \cdot \alpha(s) e^{-\int_{0}^{s} \alpha(u) \mathrm{d} u} \mathrm{~d} s<\frac{\varepsilon}{3},
\end{aligned}
$$

i.e., $\lim _{t \rightarrow \infty} P(t)=g(P(\infty))$, which imply $P(\infty)=g$ $(P(\infty))$.

Thus, Lemma 2 is proved.

It is easy to prove that the function $g(x)$ has the following properties.

\section{Lemma 3}

(1) $g(0)>0, g(1)=1$

(2) $g(x)$ is increasing on $[0,1]$

(3) $g(x)$ is a strictly concave function on $[0,1]$

Theorem 2. The extinction probability $P(\infty)$ is the smallest solution of the equation

$$
x=g(x), \quad 0 \leq x \leq 1 .
$$

Proof. By Lemma 3, $g(x)$ is a strictly concave function on $[0,1]$, and thus, $\widetilde{g}(x)=g(x)-x$ is also a strictly concave function on $[0,1]$. It is easy to see that any strictly concave function has at most two different roots in its definition domain; hence, $\widetilde{g}(x)=0$ has at most two different solutions on $[0,1]$, one of which is $x=1$. Let $x=q$ be the smallest solution of the equation $x=g(x)$.

(1) If $q=1$, since $q=1$ is the smallest solution of the equation, then the equation has no solution in $(0,1)$. But, by Lemma $2, P(\infty)$ is the solution of the equation, infer that $P(\infty)=1$, i.e., $P(\infty)$ is the smallest solution of the equation $x=g(x)$. 
(2) $0<q<1$, let $x$ such that $q<x<1$, since $q$ is the unique solution of the equation $x=g(x)$ in $(0,1)$, it is easy to see that $x>g(x)$.

It is easy to prove $\forall t>0, P(t) \leq q$. In fact, suppose contrarily $\exists t>0$, such that $P(t)>q$, then

$$
P(t)>g(P(t)) .
$$

Noting that $P(t)$ is increasing, then

$$
\begin{aligned}
P(t) & =\int_{0}^{t} e^{-\int_{0}^{s}(1-P(t-u)) \beta(u) \mathrm{d} u} \cdot \alpha(s) e^{-\int_{0}^{s} \alpha(u) \mathrm{d} u} \mathrm{~d} s \\
& \leq \int_{0}^{t} e^{-\int_{0}^{s}(1-P(t)) \beta(u) \mathrm{d} u} \cdot \alpha(s) e^{-\int_{0}^{s} \alpha(u) \mathrm{d} u} \mathrm{~d} s \\
& \leq \int_{0}^{\infty} e^{-\int_{0}^{s}(1-P(t)) \beta(u) \mathrm{d} u} \cdot \alpha(s) e^{-\int_{0}^{s} \alpha(u) \mathrm{d} u} \mathrm{~d} s \\
& =g(P(t)) .
\end{aligned}
$$

This contradicts $P(t)>g(P(t))$, so the above assumption is not true. By Lemma 2, $P(\infty)$ is the solution of the equation, i.e., $P(\infty)=g(P(\infty)) \leq q$. By the fact that $q$ is the smallest solution of the equation, imply that $P(\infty)=q$. So $P(\infty)$ is the smallest solution of the equation. Therefore, the theorem is proved.

Corollary 1. If $\beta(\cdot)=\beta>0$, then

$$
P(\infty)=\frac{\alpha}{\beta} \wedge 1 \text {. }
$$

Furthermore, $P(\infty)=1 \Leftrightarrow \alpha \geq \beta$.

Proof. If $\beta(\cdot)=\beta>0$, then

$$
\begin{aligned}
g(x) & =\int_{0}^{\infty} e^{-(1-x) \int_{0}^{s} \beta(u) \mathrm{d} u} \cdot \alpha e^{-\alpha s} \mathrm{~d} s \\
& =\int_{0}^{\infty} \alpha e^{-[(1-x) \beta+\alpha] s} \mathrm{~d} s \\
& =\frac{\alpha}{(1-x) \beta+\alpha} .
\end{aligned}
$$

Let $g(x)=x$, that is,

$$
\begin{aligned}
\frac{\alpha}{(1-x) \beta+\alpha} & =x, \\
\beta x^{2}-(\beta+\alpha) x+\alpha & =0 .
\end{aligned}
$$

Obviously, $\Delta=(\beta+\alpha)^{2}-4 \beta \alpha=(\beta-\alpha)^{2} \geq 0$; the two roots of the above equation are as follows:

$$
x_{1,2}=\frac{(\beta+\alpha) \pm|\beta-\alpha|}{2 \beta} .
$$

Then,

$$
P(\infty)=\frac{\alpha}{\beta} \wedge 1,
$$

thus,

$$
P(\infty)=1 \Leftrightarrow \alpha \geq \beta .
$$

The proof is completed.

As a consequence of Theorem 2, a sufficient condition for $P(\infty)=1$ is given.

Corollary 2. $\forall s>0$, if $\int_{0}^{s} \beta(u) d u \leq \int_{0}^{s} \alpha(u) d u$, then $P(\infty)=1$.

Proof. According to the assumptions, there is

$$
\begin{aligned}
& g(x)=\int_{0}^{\infty} e^{-(1-x) \int_{0}^{s} \beta(u) \mathrm{d} u} \cdot \alpha(s) e^{-\int_{0}^{s} \alpha(u) \mathrm{d} u} \mathrm{~d} s \\
& \geq \int_{0}^{\infty} e^{-(1-x) \int_{0}^{s} \alpha(u) \mathrm{d} u} \cdot \alpha(s) e^{-\int_{0}^{s} \alpha(u) \mathrm{d} u} \mathrm{~d} s \\
& =\frac{1}{2-x} \int_{0}^{\infty}(2-x) \alpha(s) e^{-(2-x) \int_{0}^{s} \alpha(u) \mathrm{d} u} \mathrm{~d} s \\
& =\frac{1}{2-x} \text {. }
\end{aligned}
$$

If $0<x \leq 1$ is a solution of the equation $x=g(x)$, then $x$ satisfies

$$
g(x)=x \geq \frac{1}{2-x} .
$$

Thus, $x^{2}-2 x+1 \leq 0$, i.e., $(x-1)^{2} \leq 0$, so $x=1$, deduce $P(\infty)=1$.

Corollary 2 shows that when the death rate is greater than the birth rate, the population is certainly extinct, which is intuitive.

Corollary 3. Let $\left\{G_{t}\left(\beta_{1}(\cdot), \alpha(\cdot)\right)\right\}_{t \geq 0}$ and $\left\{G_{t}\left(\beta_{2}(\cdot), \alpha\right.\right.$ $(\cdot))\}_{t \geq 0}$ be two branching tree evolutions with different birth rates and the same death rate. The corresponding extinction probabilities are denoted by $P_{1}(\infty)$ and $P_{2}(\infty)$, respectively. If $\beta_{1}(u) \geq \beta_{2}(u), u \geq 0$, then $P_{1}(\infty) \leq P_{2}(\infty)$.

Proof. $\forall 0 \leq x \leq 1, \quad g_{i}(x)=\int_{0}^{\infty} e^{-(1-x) \int_{0}^{s} \beta_{i}(u) \mathrm{d} u} \cdot \alpha(s) e^{-\int_{0}^{s}}$ $\alpha(u) \mathrm{d} u \mathrm{~d} s, i=1,2$.

If $\beta_{1}(u) \geq \beta_{2}(u), u \geq 0$, then by the definition of $g_{i}(x), i=1,2$, it is easy to see that $g_{1}(x) \leq g_{2}(x), 0 \leq x \leq 1$, and $g_{1}(x)-x \leq g_{2}(x)-x, 0 \leq x \leq 1$.

Since $g_{i}(x)-x$ is a continuous function with at least one smallest root on $[0,1](i=1,2)$, and $0<g_{1}(0) \leq g_{2}(0)$, therefore, the smallest root of $g_{1}(x)-x$ on $(0,1]$ is less than or equal to the smallest root of $g_{2}(x)-x$ on $(0,1]$, and by Theorem 2, there is

$$
P_{1}(\infty) \leq P_{2}(\infty) .
$$

The proof is completed. 
Corollary 3 implies that, for two models with the same death rate, the higher the birth rate is, the lower the extinction probability is, which is intuitive.

Corollary 4. Let $\left\{G_{t}\left(\beta(\cdot), \alpha_{1}(\cdot)\right)\right\}_{t \geq 0}$ and $\left\{G_{t}\left(\beta(\cdot) \alpha_{2}(\cdot)\right)\right\}_{t \geq 0}$ be two branching tree evolutions with different death rates but with the same birth rate. The corresponding extinction probabilities are denoted by $P_{1}(\infty)$ and $P_{2}(\infty)$, respectively, $\forall s \geq 0$, if $\int_{0}^{s} \alpha_{1}(u) d u \leq \int_{0}^{s} \alpha_{2}(u) d u$, then $P_{1}(\infty) \leq P_{2}(\infty)$.

Proof. Denote $\bar{F}_{i}(s)=1-F_{i}(s)=\mathrm{e}^{-\int_{0}^{s} \alpha_{i}(u) \mathrm{d} u}, i=1,2, \forall s \geq$ 0 , if $\int_{0}^{s} \alpha_{1}(u) \mathrm{d} u \leq \int_{0}^{s} \alpha_{2}(u) \mathrm{d} u$, then $\bar{F}_{1}(s) \geq \bar{F}_{2}(s)$.

So $\int_{0}^{\infty} A(s) \mathrm{d} F_{1}(s) \leq \int_{0}^{\infty} A(s) \mathrm{d} F_{2}(s)$ for any decreasing function $A(\cdot)$.

The corresponding functions to $g(x)$ are denoted by $g_{1}(x)$ and $g_{2}(x)$, respectively. Noting that $\mathrm{e}^{-(1-x) \int_{0}^{s} \beta(u) \mathrm{d} u}$ is a decreasing function with $s$, so by the definition of

$$
g_{i}(x)=\int_{0}^{\infty} e^{-(1-x) \int_{0}^{s} \beta(u) \mathrm{d} u} \mathrm{~d} F_{i}(s), \quad i=1,2 .
$$

imply $g_{1}(x) \leq g_{2}(x), 0 \leq x \leq 1$, then $g_{1}(x)-x \leq g_{2}(x)-x$, $0 \leq x \leq 1$,

Since $g_{i}(x)-x$ is a continuous function with at least one smallest root on $[0,1], i=1,2$, and $0<g_{1}(0) \leq g_{2}(0)$, therefore, the smallest root of $g_{1}(x)-x$ on $(0,1]$ is less than or equal to the smallest root of $g_{2}(x)-x$ on $(0,1]$, and by Theorem 2,

$$
P_{1}(\infty) \leq P_{2}(\infty)
$$

Corollary 4 shows that, for two models with the same birth rate, the randomly longer the lifespan is, the smaller the extinction probability is, which is intuitive.

Theorem 3. $P(\infty)=1 \Leftrightarrow g^{\prime}(x)<1,0<x<1$.

Proof

(1) Sufficiency: assume $g^{\prime}(x)<\underset{\sim}{1}, 0<x<1$, let $\tilde{g}(x)=g(x)-x, 0 \leq x \leq 1$, then $\widetilde{g}^{\prime}(x)=g^{\prime}(x)-1$ $<0,0<x<1$; i.e., $\widetilde{g}(x)$ is the decreasing function on $[0,1]$. Noting that $\tilde{g}(0)=g(0)>0, \tilde{g}(1)=g(1)-$ $1=0$, thus $x=1$ is the smallest root of $\tilde{g}(x)$ on $[0,1]$, and by Theorem 2 , we get $P(\infty)=1$.

(2) Necessity: assume $P(\infty)=1$, because $g(x)$ is a strictly concave function on $[0,1], \widetilde{g}(x)$ is also a strictly concave function on $[0,1]$, and $\tilde{g}(0)=g(0)>0$. In addition, by Theorem 2 and the assumptions, it is obvious that $x=1$ is the smallest root of $\widetilde{g}(x)$ on $[0,1]$, so $\widetilde{g}(x)$ is decreasing on $[0,1]$. Hence, $g^{\prime}(x)<0$, that is, $g^{\prime}(x)<1,0<x<1$.

Thus, the theorem is proved.
For $g(x)=\int_{0}^{\infty} \mathrm{e}^{-(1-x) \int_{0}^{s} \beta(u) \mathrm{d} u} \cdot \alpha(s) \mathrm{e}^{-\int_{0}^{s} \alpha(u) \mathrm{d} u} \mathrm{~d} s, 0 \leq x$ $\leq 1$ introduced above, noting that $\forall 0 \leq x \leq 1,0<g(x) \leq 1$, denote

$$
\begin{aligned}
& g_{0}(x)=x, \quad 0 \leq x \leq 1, \\
& g_{1}(x)=g(x), \\
& g_{n}(x)=g\left(g_{n-1}(x)\right), \quad n \geq 2,
\end{aligned}
$$

i.e., $g_{n}(\cdot)$ is the $n$ times iteration of $g(\cdot)$, and then, there is the following conclusion.

Theorem 4. $\forall 0 \leq x<1$, there is $\lim _{n \longrightarrow \infty} g_{n}(x)=P(\infty)$.

Proof

(1) If $P(\infty)=1$, by $g(0)>0$ and Theorem 2 , we have $\forall 0 \leq x<1, x<g(x)$. For the increasing property of $g(\cdot)$, so $g_{n}(x)<g_{n+1}(x), n \geq 1$, in addition, $g(\cdot)$ is continuous, then

$$
q=\lim _{n \longrightarrow \infty} g_{n}(x)=\lim _{n \longrightarrow \infty} g\left(g_{n-1}(x)\right)=g\left(\lim _{n \longrightarrow \infty} g_{n-1}(x)\right),
$$

i.e., $q=g(q)$, and then, we can get $q=1$ by Theorem 2, i.e., $\lim _{n \longrightarrow \infty} g_{n}(x)=P(\infty)$.

(2) If $0<P(\infty)<1, \forall 0 \leq x<P(\infty)$, then $x<g(x)$, and $g_{n}(x)<g_{n+1}(x), n \geq 1$; thus,

$$
\begin{aligned}
q & =\lim _{n \longrightarrow \infty} g_{n}(x)=\lim _{n \longrightarrow \infty} g\left(g_{n-1}(x)\right) \\
& =g\left(\lim _{n \longrightarrow \infty} g_{n-1}(x)\right)=g(q) .
\end{aligned}
$$

By Theorem 2, $q=P(\infty), \quad$ i.e., $\lim _{n \longrightarrow \infty} g_{n}(x)=P(\infty)$.

(3) If $0<P(\infty)<1, \forall P(\infty) \leq x<1$, then $x \geq g(x)$, and $g_{n}(x) \geq g_{n+1}(x), n \geq 1$, and thus,

$$
\begin{aligned}
q & =\lim _{n \longrightarrow \infty} g_{n}(x)=\lim _{n \longrightarrow \infty} g\left(g_{n-1}(x)\right) \\
& =g\left(\lim _{n \longrightarrow \infty} g_{n-1}(x)\right)=g(q) .
\end{aligned}
$$

Noting that the equation $x=g(x)$ has no root on the interval $(P(\infty), 1)$, so $q=P(\infty)$, i.e.,

$$
\lim _{n \longrightarrow \infty} g_{n}(x)=P(\infty) .
$$

Note: the significance of Theorem 4 is obvious. It gives a numerical method to calculate the asymptotic value of extinction probability. For any initial value $x_{0}\left(0 \leq x_{0}<1\right)$, iteration value $g_{n}\left(x_{0}\right)$ is the asymptotic value of the extinction probability $P(\infty)$. 


\section{The $t$-Pre-Extinction Probability}

In this section, the analytic formula and the approximation algorithm of t-pre-extinction probability are given, and the iterative integral equation with unique solution is established, which is satisfied by the t-pre-extinction probability.

Let $t>0, n \geq 1$, denote $\Delta_{n}=\left(t / 2^{n}\right)$. Divide the interval $(0, t]$ equally into $2^{n}$ intervals $\left(k \Delta_{n},(k+1) \Delta_{n}\right], k=0,1,2$, $\ldots, 2^{n}-1$. Step function is defined as follows:

$$
H_{n}(s)= \begin{cases}0, & 0 \leq s \leq \Delta_{n} \\ H_{n}\left(\Delta_{n}\right), & \Delta_{n}<s \leq 2 \Delta_{n}, \\ \cdots & \cdots \\ H_{n}\left(k \Delta_{n}\right), & k \Delta_{n}<s \leq(k+1) \Delta_{n}, \\ \cdots & \cdots \\ H_{n}\left(t-\Delta_{n}\right), & t-\Delta_{n}<s \leq t,\end{cases}
$$

where

$$
\begin{aligned}
H_{n}\left(\Delta_{n}\right)= & \int_{0}^{\Delta_{n}} e-\int_{0}^{s}\left(1-H_{n}\left(\Delta_{n}-u\right)\right) \beta(u) \mathrm{d} u \\
& \cdot \alpha(s) e^{-\int_{0}^{s} \alpha(u) \mathrm{d} u} \mathrm{~d} s ; \\
H_{n}\left(k \Delta_{n}\right)= & \int_{0}^{k \Delta_{n}} e^{-\int_{0}^{s}\left(1-H_{n}\left(k \Delta_{n}-u\right)\right) \beta(u) \mathrm{d} u} \\
& \cdot \alpha(s) e^{-\int_{0}^{s} \alpha(u) \mathrm{d} u} \mathrm{~d} s, \quad k
\end{aligned}
$$

We always assume that the birth rate function $\beta(\cdot)$ is bounded in any finite interval; denote

$$
\beta=\sup _{0 \leq u \leq t} \beta(u) .
$$

\section{Theorem 5}

(1) $\forall n \geq 1, H_{n}(\cdot)$ is nondecreasing on $[0, t]$

(2) $\left\{H_{n}(\cdot)\right\}_{n \geq 1}$ is a monotonic increasing sequence of functions

(3) $\forall t \geq 0, \lim _{n \longrightarrow \infty} H_{n}(t)=P(t)$

Proof

(1) To prove $H_{n}(\cdot)$ is a nondecreasing function on $[0, t]$ because

$$
\begin{aligned}
H_{n}\left(\Delta_{n}\right)= & \int_{0}^{\Delta_{n}} e^{-\int_{0}^{s}\left(1-H_{n}\left(\Delta_{n}-u\right)\right) \beta(u) \mathrm{d} u} \\
& \cdot \alpha(s) e^{-\int_{0}^{s} \alpha(u) \mathrm{d} u} \mathrm{~d} s \\
\geq & \int_{0}^{\Delta_{n}} \alpha(s) \mathrm{e}^{-\int_{0}^{s} \alpha(u) \mathrm{d} u} \mathrm{~d} s>0 .
\end{aligned}
$$

So, $H_{n}(\cdot)$ is nondecreasing on $\left[0,2 \Delta_{n}\right]$; suppose inductively that $H_{n}(\cdot)$ is nondecreasing on $\left[0, k \Delta_{n}\right]$, then

$$
\begin{aligned}
H_{n}\left(k \Delta_{n}\right) & =\int_{0}^{k \Delta_{n}} e^{-\int_{0}^{s}\left(1-H_{n}\left(k \Delta_{n}-u\right)\right) \beta(u) \mathrm{d} u} \cdot \alpha(s) e^{-\int_{0}^{s} \alpha(u) \mathrm{d} u} \mathrm{~d} s \\
& \geq \int_{0}^{(k-1) \Delta_{n}} e^{-\int_{0}^{s}\left(1-H_{n}\left(k \Delta_{n}-u\right)\right) \beta(u) \mathrm{d} u} \cdot \alpha(s) e^{-\int_{0}^{s} \alpha(u) \mathrm{d} u} \mathrm{~d} s \\
& \geq \int_{0}^{(k-1) \Delta_{n}} e^{-\int_{0}^{s}\left(1-H_{n}\left((k-1) \Delta_{n}-u\right)\right) \beta(u) \mathrm{d} u} \cdot \alpha(s) e^{-\int_{0}^{s} \alpha(u) \mathrm{d} u} \mathrm{~d} s \\
& =H_{n}\left((k-1) \Delta_{n}\right) .
\end{aligned}
$$

Thus, $H_{n}(\cdot)$ is nondecreasing on $\left[0,(k+1) \Delta_{n}\right]$. It is deduced by mathematical induction that $H_{n}(\cdot)$ is nondecreasing on $[0, t]$.

(2) $\forall n \geq 1$, to prove $H_{n}(\cdot) \leq H_{n+1}(\cdot)$ on $\left[0, k \Delta_{n}\right]$, $k=1,2, \ldots, 2^{n}$.

$$
\begin{aligned}
0 \leq s \leq \Delta_{n}, H_{n}(s) & =0, \\
0 & \leq s \leq \frac{\Delta_{n}}{2}=\Delta_{n+1}, H_{n+1}(s)=0, \\
\frac{\Delta_{n}}{2}<s \leq \Delta_{n} & =2 \Delta_{n+1}, H_{n+1}(s)=H_{n+1}\left(\Delta_{n+1}\right)>0 .
\end{aligned}
$$


Hence, $H_{n}(\cdot) \leq H_{n+1}(\cdot)$ on $\left[0, \Delta_{n}\right]$. Suppose inductively that $H_{n}(\cdot) \leq H_{n+1}(\cdot)$ on $\left[0, k \Delta_{n}\right]$, by the definitions of $H_{n}(\cdot)$ and $H_{n+1}(\cdot)$, we have

$H_{n}(s)=H_{n}\left(k \Delta_{n}\right), \quad k \Delta_{n}<s \leq(k+1) \Delta_{n}$,

$H_{n+1}(s)=H_{n+1}\left(2 k \Delta_{n+1}\right), \quad 2 k \Delta_{n+1}=k \Delta_{n}<s \leq k \Delta_{n}+\Delta_{n+1}$,

$H_{n+1}(s)=H_{n+1}\left((2 k+1) \Delta_{n+1}\right), \quad(2 k+1) \Delta_{n+1}<s \leq(k+1) \Delta_{n}$,

where

$$
\begin{aligned}
& H_{n+1}\left((2 k+1) \Delta_{n+1}\right) \geq H_{n+1}\left(2 k \Delta_{n+1}\right)=H_{n+1}\left(k \Delta_{n}\right) \\
& =\int_{0}^{k \Delta_{n}} e^{-\int_{0}^{s}\left(1-H_{n+1}\left(k \Delta_{n}-u\right)\right) \beta(u) \mathrm{d} u} \\
& \cdot \alpha(s) e^{-\int_{0}^{s} \alpha(u) \mathrm{d} u} \mathrm{~d} s \\
& \geq \int_{0}^{k \Delta_{n}} e^{-\int_{0}^{s}\left(1-H_{n}\left(k \Delta_{n}-u\right)\right) \beta(u) \mathrm{d} u} \\
& \cdot \alpha(s) e^{-\int_{0}^{s} \alpha(u) \mathrm{d} u} \mathrm{~d} s=H_{n}\left(k \Delta_{n}\right) .
\end{aligned}
$$

$$
\begin{array}{r}
|P(u)-P(v)|<\varepsilon, \\
|u-v|<\Delta .
\end{array}
$$

The following conclusion can be deduced by mathematical induction:

$0 \leq P(k \Delta)-H(k \Delta) \leq k \varepsilon \beta \Delta+o(\varepsilon \beta \Delta), \quad k=1,2, \ldots, 2^{n}$.

It is easy to prove that $H(k \Delta) \leq P(k \Delta), k=1,2, \ldots, 2^{n}$.

$$
\begin{aligned}
& P(\Delta)-H(\Delta)=\int_{0}^{\Delta}\left[e^{-\int_{0}^{s}(1-P(\Delta-u)) \beta(u) \mathrm{d} u}-e^{-\int_{0}^{s}(1-H(\Delta-u)) \beta(u) \mathrm{d} u}\right] \cdot \alpha(s) e^{-\int_{0}^{s} \alpha(u) \mathrm{d} u} \mathrm{~d} s \\
& =\int_{0}^{\Delta}\left[\int_{0}^{s}(P(\Delta-u)) \beta(u) \mathrm{d} u+1\right] \cdot e^{-\int_{0}^{s} \beta(u) \mathrm{d} u} \cdot \alpha(s) e^{-\int_{0}^{s} \alpha(u) \mathrm{d} u} \mathrm{~d} s \\
& \leq \int_{0}^{\Delta}[\varepsilon \beta \Delta+0(\varepsilon \beta \Delta)] \cdot \alpha(s) e^{-\int_{0}^{s} \alpha(u) \mathrm{d} u} \mathrm{~d} s
\end{aligned}
$$

$\leq \varepsilon \beta \Delta+o(\varepsilon \beta \Delta)$

$$
\begin{aligned}
& P(2 \Delta)-H(2 \Delta) \leq \int_{0}^{2 \Delta}\left[e_{0}^{s}(P(2 \Delta-u)-H(2 \Delta-u)) \beta(u) \mathrm{d} u+1\right] \cdot \alpha(s) e^{-\int_{0}^{s} \alpha(u) \mathrm{d} u} \mathrm{~d} s \\
& =\int_{0}^{\Delta}\left[\int_{0}^{s}(P(2 \Delta-u)-H(2 \Delta-u)) \beta(u) \mathrm{d} u+1\right] \cdot \alpha(s) e^{-\int_{0}^{s} \alpha(u) \mathrm{d} u} \mathrm{~d} s \\
& +\int_{\Delta}^{2 \Delta}\left[\int_{0}^{s}(P(2 \Delta-u)-H(2 \Delta-u)) \beta(u) \mathrm{d} u+1\right] \cdot \alpha(s) e^{-\int_{0}^{s} \alpha(u) \mathrm{d} u} \mathrm{~d} s .
\end{aligned}
$$


When $0 \leq s \leq \Delta$,

$$
\begin{aligned}
& \int_{0}^{s}(P(2 \Delta-u)-H(2 \Delta-u)) \beta(u) \mathrm{d} u \\
& \leq \int_{0}^{s}(P(2 \Delta-u)-P(\Delta)+P(\Delta)-H(\Delta)) \beta(u) \mathrm{d} u \\
& \leq \varepsilon \beta \Delta+o(\varepsilon \beta \Delta) \leq 2 \varepsilon \beta \Delta+o(2 \varepsilon \beta \Delta) .
\end{aligned}
$$

When $\Delta \leq s \leq 2 \Delta$,

$$
\begin{aligned}
& \int_{0}^{s}(P(2 \Delta-u)-H(2 \Delta-u)) \beta(u) \mathrm{d} u \\
& =\int_{0}^{\Delta}(P(2 \Delta-u)-H(2 \Delta-u)) \beta(u) \mathrm{d} u+\int_{\Delta}^{s}(P(2 \Delta-u) \\
& \quad-H(2 \Delta-u)) \beta(u) \mathrm{d} u \\
& \leq \varepsilon \beta \Delta+o(\varepsilon \beta \Delta)+\int_{\Delta}^{s}(P(2 \Delta-u)-P(\Delta) \\
& \quad+P(\Delta)-H(\Delta)) \beta(u) \mathrm{d} u \\
& \leq 2 \varepsilon \beta \Delta+o(2 \varepsilon \beta \Delta) .
\end{aligned}
$$

So,

$$
\begin{aligned}
& P(2 \Delta)-H(2 \Delta) \\
& \leq \int_{0}^{\Delta}(2 \varepsilon \beta \Delta+o(2 \varepsilon \beta \Delta)) \cdot \alpha(s) e^{-\int_{0}^{s} \alpha(u) \mathrm{d} u} \mathrm{~d} s+\int_{\Delta}^{2 \Delta}(2 \varepsilon \beta \Delta+o(2 \varepsilon \beta \Delta)) \cdot \alpha(s) e^{-\int_{0}^{s} \alpha(u) \mathrm{d} u} \mathrm{~d} s \\
& =\int_{0}^{2 \Delta}(2 \varepsilon \beta \Delta+o(2 \varepsilon \beta \Delta)) \cdot \alpha(s) e^{-\int_{0}^{s} \alpha(u) \mathrm{d} u} \mathrm{~d} s \\
& \leq 2 \varepsilon \beta \Delta+o(2 \varepsilon \beta \Delta) .
\end{aligned}
$$

Suppose inductively that $P(j \Delta)-H(j \Delta) \leq j \varepsilon \beta \Delta+o \quad$ Then, $(j \varepsilon \beta \Delta), 1 \leq j \leq k-1$.

$$
\begin{aligned}
& P(k \Delta)-H(k \Delta) \leq \int_{0}^{k \Delta}\left[\int_{0}^{s}(P(k \Delta-u)-H(k \Delta-u)) \beta(u) \mathrm{d} u-1\right] \cdot \alpha(s) e^{-\int_{0}^{s} \alpha(u) \mathrm{d} u} \mathrm{~d} s \\
& =\sum_{j=0}^{k-1} \int_{j \Delta}^{(j+1) \Delta}\left[e_{0}^{s}(P(k \Delta-u)-H(k \Delta-u)) \beta(u) \mathrm{d} u-1\right] \cdot \alpha(s) e^{-\int_{0}^{s} \alpha(u) \mathrm{d} u} \mathrm{~d} s .
\end{aligned}
$$


When $(k-1) \Delta \leq s \leq k \Delta$,

$$
\begin{aligned}
& \int_{0}^{s}(P(k \Delta-u)-H(k \Delta-u)) \beta(u) \mathrm{d} u \\
& =\sum_{j=0}^{k-2} \int_{j \Delta}^{(j+1) \Delta}(P(k \Delta-u)-H(k \Delta-u)) \beta(u) \mathrm{d} u+\int_{(k-1) \Delta}^{s}(P(k \Delta-u)-H(k \Delta-u)) \beta(u) \mathrm{d} u,
\end{aligned}
$$

$\forall 0 \leq j \leq k-2$,

$$
\begin{aligned}
& \int_{j \Delta}^{(j+1) \Delta}(P(k \Delta-u)-H(k \Delta-u)) \beta(u) \mathrm{d} u \\
& =\int_{j \Delta}^{(j+1) \Delta}[P(k \Delta-u)-P((k-j-1) \Delta)+P((k-j-1) \Delta)-H((k-j-1) \Delta)] \beta(u) \mathrm{d} u \\
& <\varepsilon \beta \Delta+o(\varepsilon \beta \Delta), \\
& \int_{(k-1) \Delta}^{s}(P(k \Delta-u)-H(k \Delta-u)) \beta(u) \mathrm{d} u \leq \varepsilon \beta \Delta+o(\varepsilon \beta \Delta) .
\end{aligned}
$$

So

$\int_{0}^{s}(P(k \Delta-u)-H(k \Delta-u)) \beta(u) \mathrm{d} u \leq k \varepsilon \beta \Delta+o(k \varepsilon \beta \Delta)$.

It is obvious that when $s_{1} \leq s_{2}$, there is

$$
\begin{aligned}
& \int_{0}^{s_{1}}(P(k \Delta-u)-H(k \Delta-u)) \beta(u) \mathrm{d} u \\
& \quad \leq \int_{0}^{s_{2}}(P(k \Delta-u)-H(k \Delta-u)) \beta(u) \mathrm{d} u .
\end{aligned}
$$

So

$$
\begin{aligned}
& P(k \Delta)-H(k \Delta) \\
& \leq \sum_{j=0}^{k-1} \int_{j \Delta}^{(j+1) \Delta}[k \varepsilon \beta \Delta+o(k \varepsilon \beta \Delta)] \cdot \alpha(s) e^{-\int_{0}^{s} \alpha(u) \mathrm{d} u} \mathrm{~d} s \\
& =\int_{0}^{k \Delta}[k \varepsilon \beta \Delta+o(k \varepsilon \beta \Delta)] \cdot \alpha(s) e^{-\int_{0}^{s} \alpha(u) \mathrm{d} u} \mathrm{~d} s \\
& \leq k \varepsilon \beta \Delta+o(k \varepsilon \beta \Delta) .
\end{aligned}
$$

It is proved deductively that $0 \leq P(k \Delta)-H(k \Delta) \leq$ $k \varepsilon \beta \Delta+o(k \varepsilon \beta \Delta), k=1,2, \ldots, 2^{n}$.

Especially, $0 \leq P(t)-H(t) \leq \varepsilon \beta t+o(\varepsilon \beta t)$.

That is,

$$
\lim _{n \longrightarrow \infty} H_{n}(t)=P(t) .
$$

The proof is complete.

Theorem 6. The t-pre-extinction probability $P(t)$ is the unique solution of the iterative integral equation in Theorem 1.
Proof. According to Theorem 1, the t-pre-extinction probability is the solution of the iterative integral equation in Theorem 1, and it is not difficult to deduce by Theorem 5 that the solution of the iterative integral equation is unique. Therefore, the t-pre-extinction probability is the unique solution of the iterative integral equation. The theorem is proved.

\section{The Stochastic Order of Extinction Moment}

If $P(\infty)=1$, then the extinction moment $T(\omega)$ is a realvalued random variable, and the t-pre-extinction probability $P(t)$ is the distribution function of $T(\omega)$. In this section, we study the stochastic order of the extinction moment for different branching tree evolutions.

\section{Theorem 7}

(1) Let $P_{1}(\infty)$ and $T_{1}(\omega)$ be, respectively, the extinction probability and extinction moment for the branching tree evolutions $\left\{G_{t}\left(\beta_{1}(\cdot), \alpha(\cdot)\right)\right\}_{t \geq 0}$; let $P_{2}(\infty)$ and $T_{2}(\omega)$ be, respectively, the extinction probability and extinction moment for the branching tree evolutions $\left\{G_{t}\left(\beta_{2}(\cdot), \alpha(\cdot)\right)\right\}_{t \geq 0}$. If $P_{1}(\infty)=1, P_{2}(\infty)=1$, and $\beta_{1}(\cdot) \leq \beta_{2}(\cdot)$, then $T_{1}(\omega)$ is stochastically smaller than $T_{2}(\omega)$, that is $T_{1}(\omega) \leq$ s.t. $T_{2}(\omega)$.

(2) Let $\widetilde{P}_{1}(\infty)$ and $\widetilde{T}_{1}(\omega)$ be, respectively, the extinction probability and extinction moment for the branching tree evolutions $\left\{G_{t}\left(\beta(\cdot), \alpha_{1}(\cdot)\right)\right\}_{t \geq 0}$; let $\widetilde{P}_{2}(\infty)$ and $\widetilde{T}_{2}(\omega)$ be, respectively, the extinction probability and extinction moment for the branching tree evolutions $\left\{G_{t}\left(\beta(\cdot), \alpha_{2}(\cdot)\right)\right\}_{t \geq 0}$. If $\widetilde{P}_{1}(\infty)=1, \widetilde{P}_{2}(\infty)=1$, and $\forall t>0, \int_{0}^{t} \alpha_{1}(s) d s \leq \int_{0}^{t} \alpha_{2}(s) d s$, then $\widetilde{T}_{2}(\omega)$ is stochastically smaller than $\widetilde{T}_{1}(\omega)$, that is $\widetilde{T}_{2}(\omega) \leq^{\text {s.t. }} \widetilde{T}_{1}(\omega)$. 
Proof

(1) Corresponding to the branching tree evolution $\left\{G_{t}\left(\beta_{1}(\cdot), \alpha(\cdot)\right)\right\}_{t \geq 0}$ and $\left\{G_{t}\left(\beta_{2}(\cdot), \alpha(\cdot)\right)\right\}_{t \geq 0}$, similarly to Theorem 5 , define the step function series as $\left\{H_{n}^{(1)}(\cdot)\right\}_{n \geq 1}$ and $\left\{H_{n}^{(2)}(\cdot)\right\}_{n \geq 1}$, respectively.

By the hypothesis of $\beta_{1}(\cdot) \leq \beta_{2}(\cdot)$ and the definition of $H_{n}^{(i)}(\cdot), i=1,2$, applying the mathematical induction, it is easy to prove that $H_{n}^{(1)}\left(k \Delta_{n}\right) \geq H_{n}^{(2)}\left(k \Delta_{n}\right), 1 \leq k \leq 2^{n}, n \geq 1$, and thus $H_{n}^{(1)}(\cdot) \geq H_{n}^{(2)}(\cdot)$. By Theorem 5,

$P_{1}(t)=P\left(T_{1}(\omega) \leq t\right)=\lim _{n \longrightarrow \infty} H_{n}^{(1)}(t), \quad t \geq 0$,

$P_{2}(t)=P\left(T_{2}(\omega) \leq t\right)=\lim _{n \longrightarrow \infty} H_{n}^{(2)}(t), \quad t \geq 0$.

Thus, $P_{1}(t) \geq P_{2}(t), t \geq 0$.

Because $T_{1}(\omega) \leq$ s.t. $T_{2}(\omega) \Leftrightarrow P_{1}(t) \geq P_{2}(t), t \geq 0$, so $T_{1}(\omega) \leq^{\text {s.t. }} T_{2}(\omega)$. Thus, $(1)$ is proved.

(2) Corresponding to the branching tree evolution $\left\{G_{t}\left(\beta(\cdot), \alpha_{1}(\cdot)\right)\right\}_{t \geq 0}$ and $\left\{G_{t}\left(\beta(\cdot), \alpha_{2}(\cdot)\right)\right\}_{t \geq 0}$, similarly to Theorem 5 , define the step function series as $\left\{\tilde{H}_{n}^{(1)}(\cdot)\right\}_{n \geq 1}$ and $\left\{\tilde{H}_{n}^{(2)}(\cdot)\right\}_{n \geq 1}$, respectively, and denote $F_{i}(t)=1-\mathrm{e}^{-\int_{0}^{t} \alpha_{i}(u) \mathrm{d} u}, t \geq 0, i=1,2$; then $F_{1}(t) \leq F_{2}(t), \quad 0 \leq t<\infty$. Denote $D_{i}(s)=I_{\left[0, k \triangle_{n}\right]}$ $(s) \mathrm{e}^{-} \quad \int_{0}^{s}\left(1-\widetilde{H}_{n}^{(i)}\left(k \triangle_{n}-u\right)\right) \beta(u) \mathrm{d} u, s \longrightarrow 0, i=$ 1,2 ; then $D_{i}(s)$ is a decreasing function of $s$. Applying the mathematical induction, we have

$$
\begin{aligned}
\tilde{H}_{n}^{(1)}\left(k \triangle_{n}\right) & =\int_{0}^{k \triangle_{n}} e^{-\int_{0}^{s}\left(1-\widetilde{H}_{n}^{(i)}\left(k \triangle_{n}-u\right)\right) \beta(u) \mathrm{d} u} \mathrm{~d} F_{1}(s) \\
& =\int_{0}^{\infty} D_{1}(s) \mathrm{d} F_{1}(s) \leq \int_{0}^{\infty} D_{1}(s) \mathrm{d} F_{2}(s) \\
& \leq \int_{0}^{\infty} D_{2}(s) \mathrm{d} F_{2}(s)=\widetilde{H}_{n}^{(2)}\left(k \triangle_{n}\right), \quad 1 \leq k \leq 2^{n} .
\end{aligned}
$$

Thus, $\widetilde{H}_{n}^{(1)}(\cdot) \leq \widetilde{H}_{n}^{(2)}(\cdot)$, and by Theorem 5 ,

$$
\begin{aligned}
& \widetilde{P}_{1}(t)=P\left(\widetilde{T}_{1}(\omega) \leq t\right)=\lim _{n \longrightarrow \infty} \widetilde{H}_{n}^{(1)}(t), \quad t \geq 0, \\
& \widetilde{P}_{2}(t)=P\left(\widetilde{T}_{2}(\omega) \leq t\right)=\lim _{n \longrightarrow \infty} \widetilde{H}_{n}^{(2)}(t), \quad t \geq 0 .
\end{aligned}
$$

Then, $\widetilde{P}_{1}(t) \leq \widetilde{P}_{2}(t)$, so $\widetilde{T}_{1}(\omega) \geq^{\text {s.t. }} \widetilde{T}_{2}(\omega)$. Thus, (2) is proved and theorem is proved.

\section{Conclusions}

This paper addresses an important problem in the field of branching process. The extinction probability and the t-preextinction probability are studied by constructing a branching tree evolution model in which the birth rate and the death rate are both dependent on node's age. The analytical formula and the approximation algorithm for the distribution of extinction moment are given; furthermore, the analytical formula and the approximation algorithm of extinction probability are given, and a necessary and sufficient condition of extinction with probability 1 is given.

Due to publishing constraints, only the population extinction is studied, the graph-topological properties and the age structure of nodes will be studied in subsequent papers.

\section{Data Availability}

No data were used to support the findings of this study.

\section{Conflicts of Interest}

The authors declare that they have no conflicts of interest.

\section{Acknowledgments}

This study was supported by Shanghai Natural Science Foundation (no. 16ZR1414000).

\section{References}

[1] T. E. Harris, The Theory of Branching Processes, SpringerVerlag, Berlin, Germany, 1963.

[2] W. L. Smith and W. E. Wilkinson, "On branching processes in random environments," The Annals of Mathematical Statistics, vol. 40, no. 3, pp. 814-827, 1969.

[3] H.-X. Wang and D. Fang, "Asymptotic behaviour of population-size-dependent branching processes in Markovian random environments," Journal of Applied Probability, vol. 36, no. 02, pp. 611-619, 1999.

[4] H.-x. Wang, "Extinction of population-size-dependent branching processes in random environments," Journal of Applied Probability, vol. 36, no. 01, pp. 146-154, 1999.

[5] P. Haccou, P. Jagers, and V. A. Vatubin, "Branching processes: variation, growth, and extinction of populations," in Cambridge Studies in Adaptive DynamicsCambridge University Press, Cambridge, UK, 2005.

[6] R. Bellman and T. Harris, "On age-dependent binary branching processes," The Annals of Mathematics, vol. 55, no. 2, pp. 280-295, 1952.

[7] J. Peter and F. C. Klebaner, "Population-size-dependent and age-dependent branching processes," Stochastic Processes and Their Applications, vol. 87, pp. 235-254, 2000.

[8] C. D. Greenman, "A path integral approach to age dependent branching processes," Journal of Statistical Mechanics: Theory and Experiment, vol. 2017, Article ID 033101, 2017.

[9] D. Kajunguri, E. B. Are, and J. W. Hargrove, "Improved estimates for extinction probabilities and times to extinction for populations of tsetse (glossing spp)," PLoS Neglected Tropical Diseases, vol. 13, no. 4, Article ID e0006973, 2019.

[10] D. Anna and F. Vadillo, "Extinction-time for stochastic population models," Journal of Computational and Applied Mathematics, vol. 295, pp. 159-169, 2016.

[11] O. Aydogmus, "On extinction time of a generalized endemic chain-binomial model," Mathematical Biosciences, vol. 279, pp. 38-42, 2016. 